\title{
DESIGN OF TEXTUAL PRESENTATION FROM ONLINE INFORMATION USING HYBRID APPROACHES
}

\author{
S. Saraswathi \\ Department of Information Technology, Pondicherry Engineering College, Pondicherry, India \\ E-mail: swathi@pec.edu
}

\begin{abstract}
This paper describes the design of online textual presentation tool developed by using various approaches like information retrieval, information extraction, topic identification and sentence weightage algorithms. To design a presentation for the given user query, the following approaches are used: Information extraction based on Boolean and probabilistic approaches, Topic identification based on semantic based approach, Sentence weightage algorithm based on importance of the terms and topics involved in the documents. A combination of these approaches led to the design of PowerPoint presentation file.
\end{abstract}

Keywords:

Recall, Precision, Textual presentation, Topic Identification, Sentence Weightage, Summarization

\section{INTRODUCTION}

Information retrieval is the science and art of locating and obtaining documents based on information needs expressed to a system in a query language. The Boolean retrieval is the most simple of these retrieval methods and relies on the use of Boolean operators. The terms are linked together using AND, OR and NOT operators to the user query. A comparison of the Boolean and probabilistic information retrieval systems was done by Robert M.Losee [1]. The approach of comparing Boolean and term weightage models has become possible because of the development of analytic models of information retrieval performances. A probabilistic system ranks the documents for retrieval by assigning a numeric value to each document, based on the weights for query terms and the frequencies of the terms occurring in the documents. The models of probabilistic retrieval [2] provides researchers with a decision rule stating that a document should be retrieved if a calculated value that is based on several parameters is less than the cost based value. A text partition model [3] was proposed to determine the boundaries of discourse structures. It was based on association of noun-noun relations and noun-verb relations defined on discourse level and sentence level respectively. Three factors are considered for topic identification:1) repetition of words, 2) importance of words and 3) collocation semantics. A window was moved from the first sentence to the last sentence and the association norms for sentences in the current window were calculated. Hierarchically organizing data [4] according to topics has been accepted as a very successful method for organizing or browsing a large volume of textual documents in information systems. Such a topic hierarchy is used as a way of systematically organizing a large document collection; incoming documents (ie. Extracted from web) are indexed on topic hierarchies, which are presented as browsable directories to users with information needs. Construction of topic hierarchies is of great importance in information systems to organize huge numbers of online text documents. Youngjoong Ko [5] has described a method to improve the text categorization using the importance of sentences. The features from important sentences should be given weightage. The authors used two kinds of text summarization techniques: one uses the title and the other uses the importance of terms. This paper discusses on the design of a textual presentation application from online information using the various techniques related to information retrieval, topic identification, and sentence weightage.

This paper is structured as follows: section 2 and section 3 provides the overall design and module description of the online presentation, section 4 and 5 discusses on results and future enhancements.

\section{DESIGN OF ONLINE PRESENTATION SYSTEM}

Different techniques exist for information retrieval, topic identification and sentence weightage methods [6]-[9]. In the proposed system the combination of these approaches are used for designing a textual presentation tool. Fig. 1 shows the overall design of online presentation and the steps needed for the design are as follows:

The user query will be send to the search engine. Documents related to the query in various file formats are collected and converted to text files. The contents of text files are checked for relevant information using information extraction techniques based on Boolean and probabilistic approaches. The topics are identified for the relevant text files using semantic based approach. Based on the sentence weightage, the presentation file is generated to the user based on the number of slides he/she requires in the Software Engineering domain.

\section{MODULE DESCRIPTION}

\subsection{COLLECTION OF RELEVANT DOCUMENTS}

The block diagram for collecting the documents is shown in the Fig.2. The user sends the required query to the search engine. The search engine will retrieve the information related to the query through the WebConversation and WebResponse classes in HttpUnit package. HttpUnit is a set of classes for automating browser functions. HttpUnit is a free, open source Java API for accessing web sites without a browser. The three main classes in the HttpUnit packages are WebConversation, WebResponse and WebRequest. The center of HttpUnit is the WebConversation class, which takes the place of a browser talking to a single site. The user has to send the needed query through WebRequest and file type to download. WebRequest is the abstract base class for the .NET framework's request or 


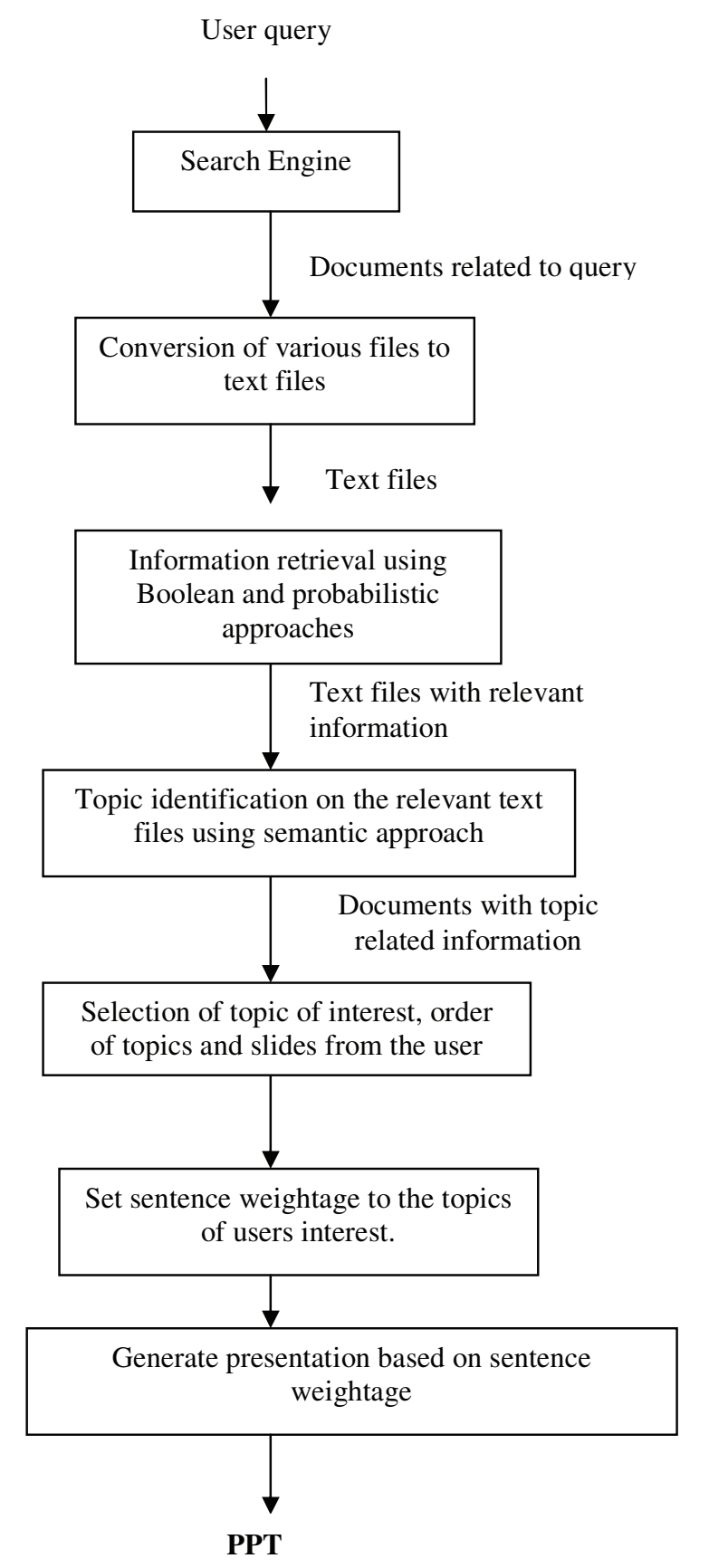

Fig.1. Block diagram of online presentation

response model for accessing data from the internet. Web response is defined as a response to a web request from a web server. In the requested page the needed information and the file type are searched and retrieved. The retrieved files in various formats are stored in the user specified folder.

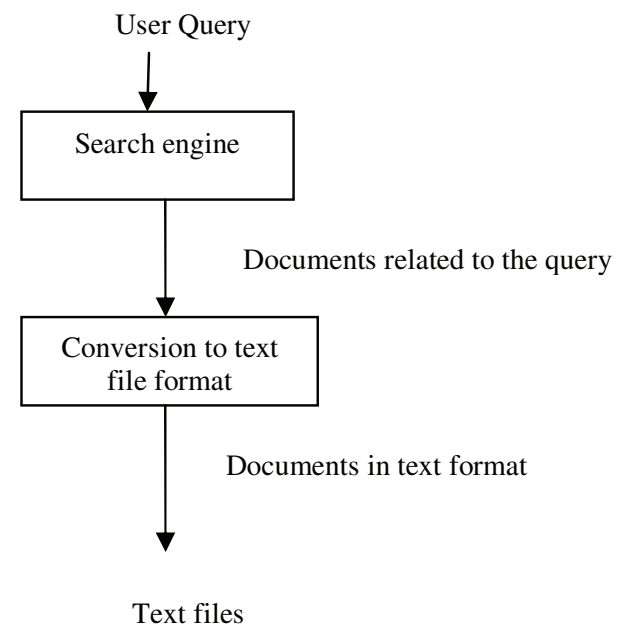

Fig.2. Block diagram for collecting documents

In order to avoid the complexity of extracting information from various file formats, all the files should be converted to text file format. To convert various file formats to text file format, the tool named convert doc is used. Convert doc tool converts file formats like DOC, HTML, RTF, PDF, to TXT format. By increasing the search page number in google, the number of downloaded documents can be increased. Converted text files are downloaded in the user specified folder.

\subsection{ELIMINATING IRRELEVANT INFORMATION}

The block diagram for eliminating the irrelevant information is shown in the Fig.3. The converted text files might contain irrelevant information like references, acknowledgement, etc.

\section{Text files}

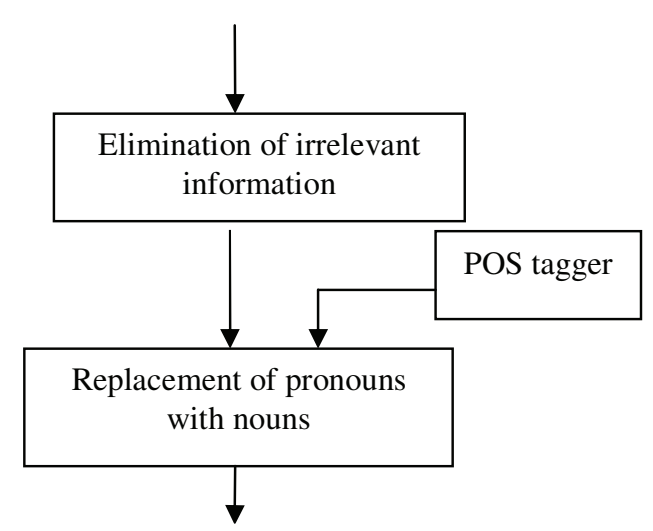

Text files with relevant information

Fig.3. Block diagram for eliminating irrelevant information

The irrelevant information is removed by detecting the keywords like acknowledgement and references. The garbage data which follows under the diagram are to be removed by detecting the keyword figure. 
The authors with their affiliations part are removed by detecting the keyword references. The regular expression in java is used to compare the keywords in this module. A regular expression is a pattern of characters that describes a set of strings. Regular expression matching also allows to test whether a string fits into a specific syntactic form, such as an email address. The information which follows under the keyword references is deleted. The text files with relevant information are tagged using the Wintree POS tagger [10]. In the tagged file the pronouns of a sentence are replaced by the corresponding noun. The resulting text file of this module contains only relevant information.

\subsection{TOPIC ASSIGNMENT}

The block diagram for topic assignment is shown in the Fig.4. To assign the topic for each paragraph the maximum occurrence of keywords (nouns and verbs) and their semantically equivalent words is determined. The maximum occurred keywords are determined. The topics related to Software Engineering are stored in a database. The maximum occurred keywords in the paragraphs are compared with the topics stored in database to assign the suitable topics for them. Paragraphs with similar topics available in different text file are grouped together.

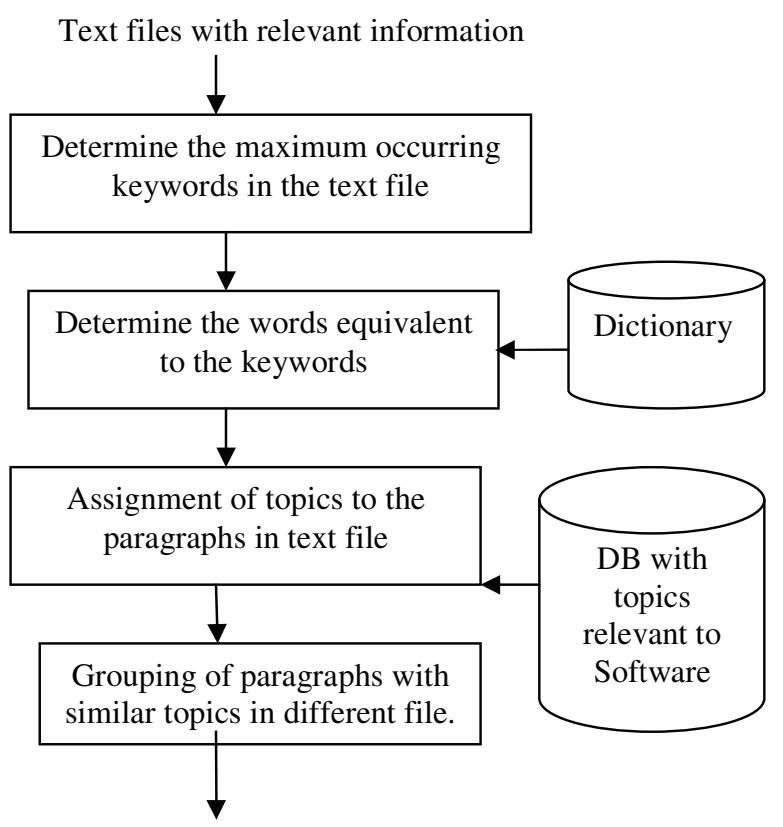

Topic assigned information

Fig.4. Block diagram for topic assignment

The next step deals with removal of semantically related sentences, within each topic assigned information. The percentage of similarity among sentences is calculated based on Euclidean distance measure of the keywords and the semantically related words present in them. If the comparison percentage exceeds the threshold value $(70 \%)$, then the sentences are considered to be similar. The matched sentence will be removed. The size of the file gets reduced after the removal of the semantically related sentences.

\subsection{GENERATION OF PPT}

The topics and the sentences related to each topic are listed to the user with the line number. The user can list the topic order for generating the presentation. He can also list the order for arranging the sentences within each topic. Based on the user's interest the topics and their contents are re-ordered. The screenshot for user interface for PPT generation is shown in Fig.5. The number of the slides and the number of the sentences per slide is given by the user. The generated PPT file is shown in Fig.6.

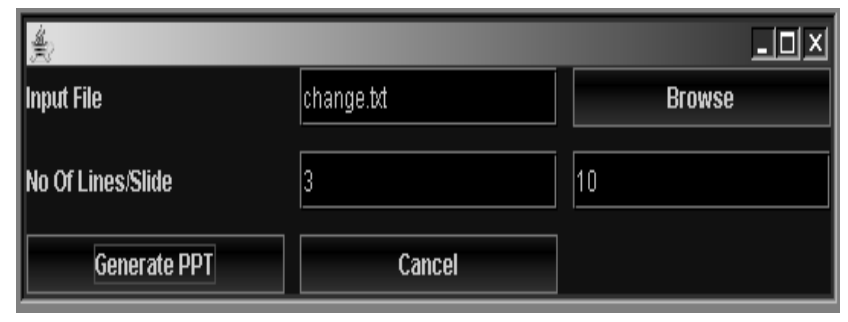

Fig.5. Screenshot for user interface for PPT generation

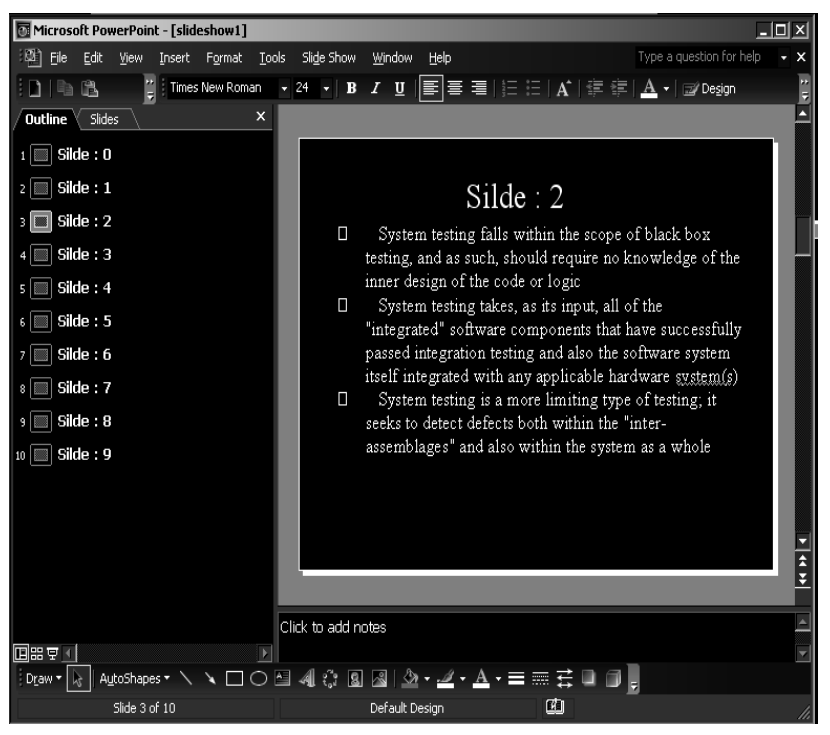

Fig.6. Generation of PPT

\section{RESULTS AND DISCUSSION}

Precision and Recall are two widely used measures for evaluating the quality of results. Precision is defined as the fraction of the retrieved sentences that are relevant, and Recall is defined as the fraction of relevant sentences that are retrieved. The hash table is used to store the most relevant and irrelevant sentence based on the users interest in preparing the presentation. The precision and recall is calculated by using the equations (1) and (2).

Precision $=\frac{\text { No.of relevant sentence retreived }}{\text { No.of sentence retreived }}$ 


\section{No.of relevant sentence retreived \\ Recall $=\frac{\text { based on users interest }}{\text { No.of relevant sentence retreived }}$}

The result is analyzed for the Software Engineering domain. Software Engineering consists of the following topics namely Introduction to Software Engineering, Process models, System engineering, Design engineering, Project scheduling, Software testing, Requirements engineering, Software metrics, Web engineering, Component based software development, etc.. The topics that are chosen for the analysis of result are Software testing, Software metrics and Component based software development (CBSD).
The number of documents that are collected for Software Engineering was 100, out of which 20 documents were relevant to the software testing topic. The average size of those 20 documents is $53 \mathrm{~KB}$. The number of topics assigned for the 20 relevant documents is 40 . The following are the some of the topics assigned in Software testing: System testing, Software testing, Integration testing, Black-box testing, White-box testing, Fault based testing, etc. The topics that are chosen for analysis are Unit testing, Acceptance testing, Performance testing, System testing.

Table.1. Precision and recall values for three domains

\begin{tabular}{|c|c|c|c|c|c|c|c|}
\hline \multirow{2}{*}{ Domain Name } & \multirow{2}{*}{$\begin{array}{l}\text { No.of } \\
\text { relevant } \\
\text { doc } \\
\text { retrieved }\end{array}$} & \multirow{2}{*}{$\begin{array}{l}\text { Name of the } \\
\text { Topic }\end{array}$} & \multirow{2}{*}{$\begin{array}{c}\text { No. of } \\
\text { Sentences } \\
\text { relevant to the } \\
\text { topic }\end{array}$} & \multicolumn{2}{|c|}{ User 1} & \multicolumn{2}{|c|}{ User 2} \\
\hline & & & & Precision & Recall & Precision & Recall \\
\hline \multirow{4}{*}{ Software testing } & \multirow{4}{*}{20} & Unit testing & 40 & 0.75 & 0.91 & 0.8 & 0.75 \\
\hline & & Acceptance testing & 42 & 0.75 & 0.8 & 0.7 & 0.75 \\
\hline & & Performance testing & 44 & 0.92 & 0.54 & 0.6 & 0.8 \\
\hline & & System testing & 45 & 0.8 & 0.68 & 0.7 & 0.75 \\
\hline \multirow{4}{*}{$\begin{array}{l}\text { Software } \\
\text { metrics }\end{array}$} & \multirow{4}{*}{20} & Software quality metrics & 40 & 0.9 & 0.61 & 0.85 & 0.7 \\
\hline & & Defect density metrics & 40 & 0.8 & 0.43 & 0.75 & 0.8 \\
\hline & & Customer problem metric & 42 & 0.8 & 0.78 & 0.8 & 0.68 \\
\hline & & Object oriented metrics & 44 & 0.91 & 0.6 & 0.6 & 0.8 \\
\hline \multirow{3}{*}{$\begin{array}{l}\text { Component- } \\
\text { based software } \\
\text { development }\end{array}$} & \multirow{3}{*}{20} & Domain engineering & 42 & 0.8 & 1.0 & 0.92 & 0.8 \\
\hline & & Component reuse & 40 & 0.8 & 1.0 & 1.0 & 0.77 \\
\hline & & $\begin{array}{l}\text { Overview of component based } \\
\text { software development }\end{array}$ & 44 & 0.8 & 0.65 & 0.65 & 0.8 \\
\hline
\end{tabular}

The number of documents that were relevant to the software metrics topic was 20. The average size of those 20 documents is $51 \mathrm{~KB}$. The number of topics assigned for the 20 relevant documents is 37 . The following are the some of the topics assigned in Software metrics: Software quality metrics, Product quality metrics, Function-based metrics, Operation-oriented metrics, Customer problem metrics, etc. The topics that are chosen for analysis are Software quality metrics, Defect density metrics, Customer problem metrics, Object oriented metrics.

The number of documents that were relevant to the CBSD is 20. The average size of those 20 documents is $48 \mathrm{~KB}$. The number of topics assigned for the 20 relevant documents is 23 . The following are the some of the topics assigned in CBSD: Overview of CBSD, Domain engineering, Component adaptation, Component qualification, Component engineering, etc. The topics that are chosen for analysis are Overview of CBSD, Domain engineering, Component reuse.

Queries related to the above topics were given as input by the users. The sentences related topics were grouped together based on several topics. The order for presenting the presentation is provided by the user. The quality of the documents is measured by using precision and recall as shown in Table 1.

The result is tested with 50 users. The users can generate the presentation based on their order of interest. Thus the variation in the precision and recall values may occur by generating the presentation on their interests. The precision and recall values are plotted for the $80 \%$ of the user as shown in the Fig.7, 8 and 9 for the various topics in Software Engineering. The remaining 


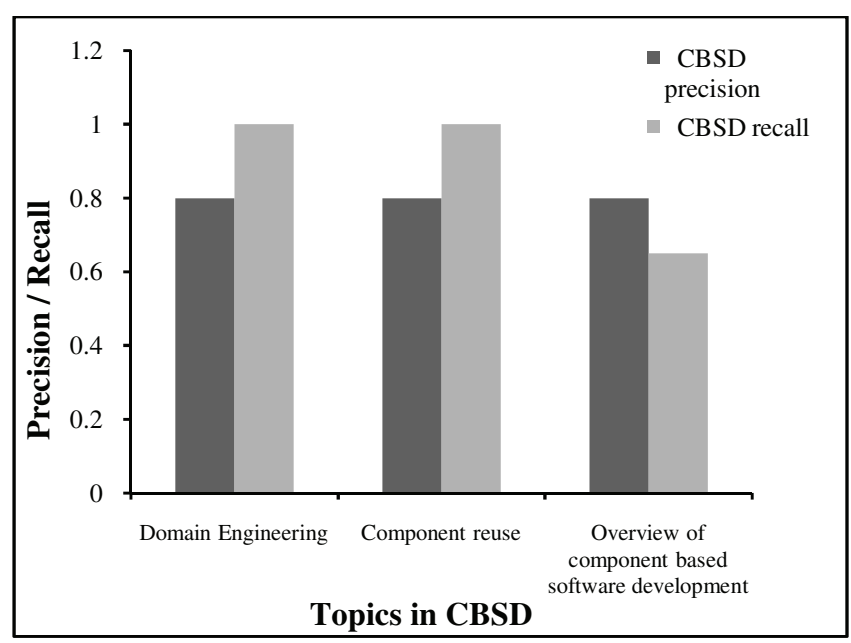

Fig.9. Precision \& recall graph for $80 \%$ of users (user1) in the topic Component Based System Design

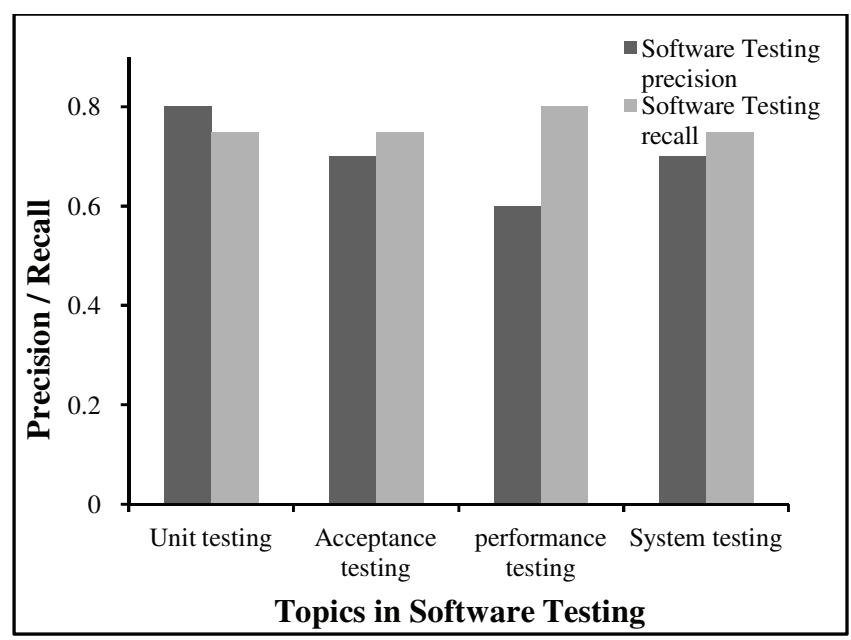

Fig.10. Precision \& recall graph for $20 \%$ of users (user2) in the topic Software Testing

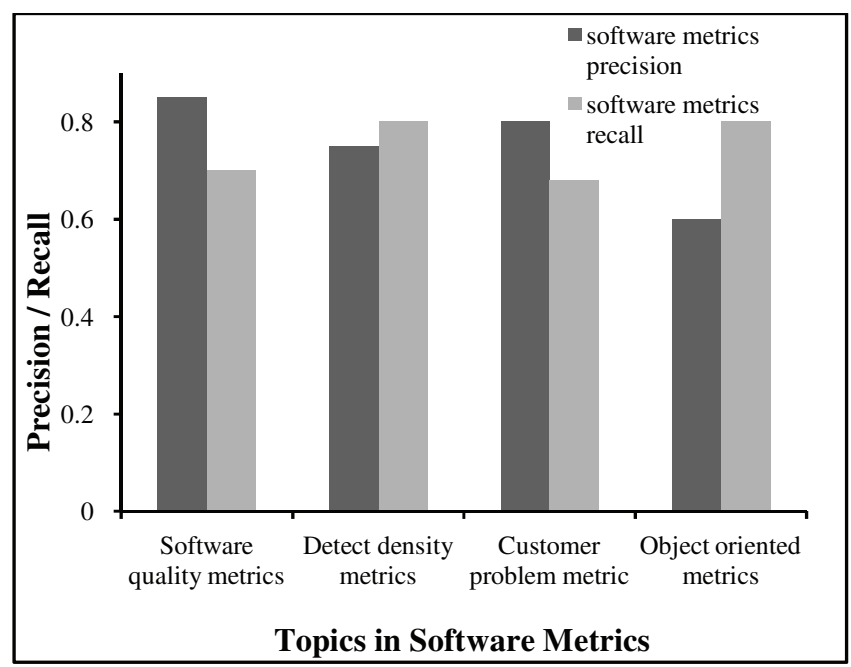

Fig.11. Precision \& recall graph for $20 \%$ of users (user2) in the topic Software Metrics 


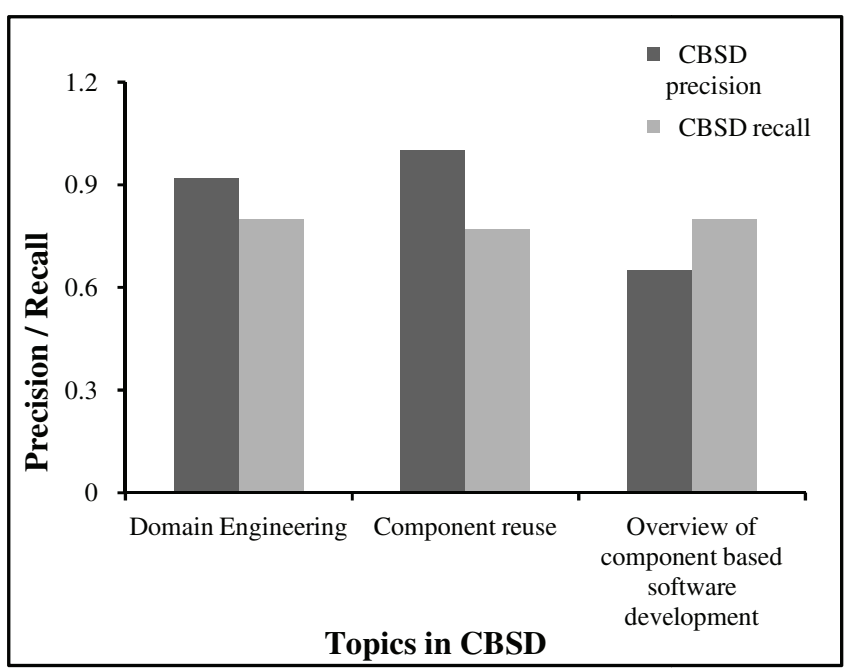

Fig.12. Precision \& recall graph for $20 \%$ of users (user2) in the topic Component Based System Design

\subsection{SOFTWARE TESTING}

The four topics plotted in this domain are unit testing, acceptance testing, performance testing and system testing. The precision and recall value for unit testing to about $80 \%$ of the users varies by less difference, and the recall value is greater than the precision, as the topic contains more number of most relevant sentences. Acceptance testing also shows the same variation for user1. Performance testing shows a large variation with respect to precision and recall for user1. The precision value is greater than a recall since it contains relevant sentences count greater than the most relevant sentences. The system testing showed less variation in between unit testing and performance testing topics for user1. The precision value is greater than a recall since it contains relevant sentences count greater than the most relevant sentences. The relevancy can be increased by adding more relevant documents. The average precision and recall for user 1 for the topic software testing is 0.8 and 0.73 . The average precision and recall for user2 for the topic software testing is 0.7 and 0.76 . The PPT generated by the users in their order of interests for the software testing is shown in the Fig. 13 and 14.

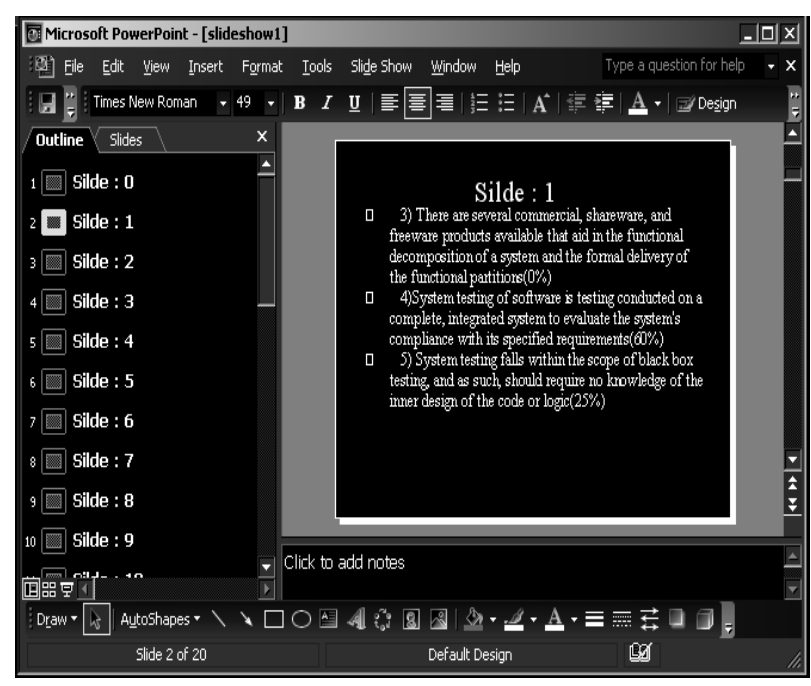

Fig.13. PPT generated by user 1 for the Software Testing topic

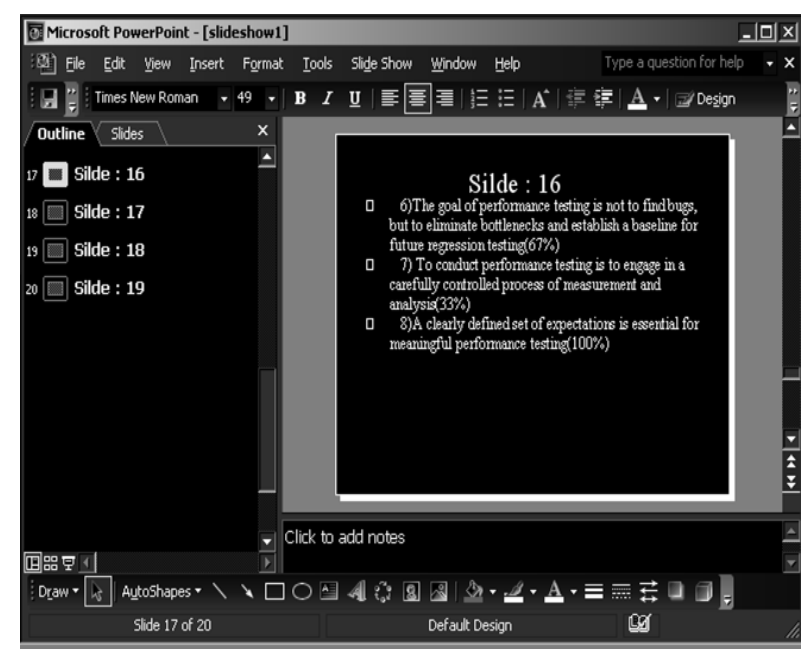

Fig.14. PPT generated by user2 for the Software Testing topic

\subsection{SOFTWARE METRICS}

The four topics plotted in this domain are software quality metrics, defect density metrics, customer problem metrics, and object oriented metrics. The software quality metrics shows a large variation with respect to precision and recall for user 1 . The precision value is greater than a recall since it contains relevant sentences count greater than the most relevant sentences. The defect density metrics also shows the same variation as software quality metrics for user1. The Customer problem metrics shows less variation for user1 since it contains slightly more number of relevant sentences. Object oriented metrics shows the precision value is greater than the recall values for user1 because of the more number of relevant sentences. The average precision and recall for user 1 for the topic software metrics is 0.86 and 0.61 . The average precision and recall for user 2 for the topic software metrics is 0.75 and 0.75 . The PPT generated by the users in their order of interests for the software metrics are shown in the Fig. 15 and 16. 


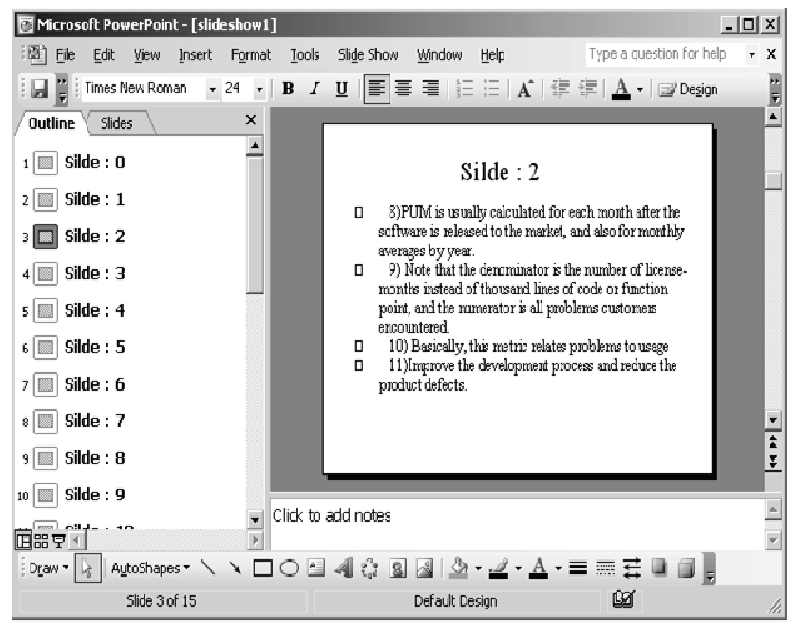

Fig.15. PPT generated by user1 for the Software Metrics topic

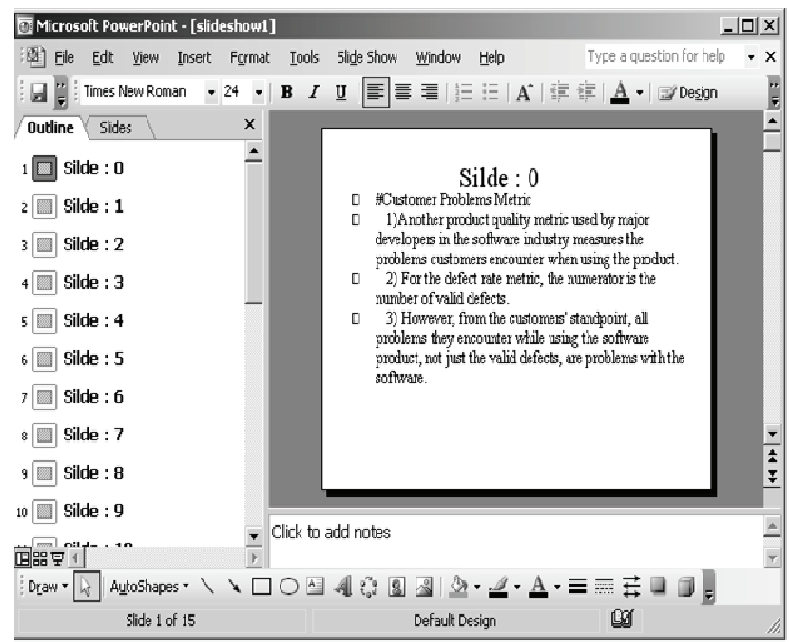

Fig.16. PPT generated by user2 for the Software Metrics topic

\subsection{COMPONENT BASED SOFTWARE DEVELOPMENT (CBSD)}

The topics plotted in this domain are domain engineering, component reuse and overview of CBSD. The first two domains contain same precision and recall values for user1. The recall value for the first two domains is 1 and it shows that most of the sentences are most relevant and only very few sentences are irrelevant. The first two topics contain no relevant sentences. Whereas for the third topic the relevant sentences and irrelevant sentences count are equal to the most relevant sentences count and hence it contains less recall value.

The average precision and recall for user1 for the topic CBSD is 0.8 and 0.88 . The average precision and recall for user2 for the topic CBSD is 0.85 and 0.79 . The PPT generated by the users in their order of interests for the CBSD topic are shown in the below Fig.17 and 18.

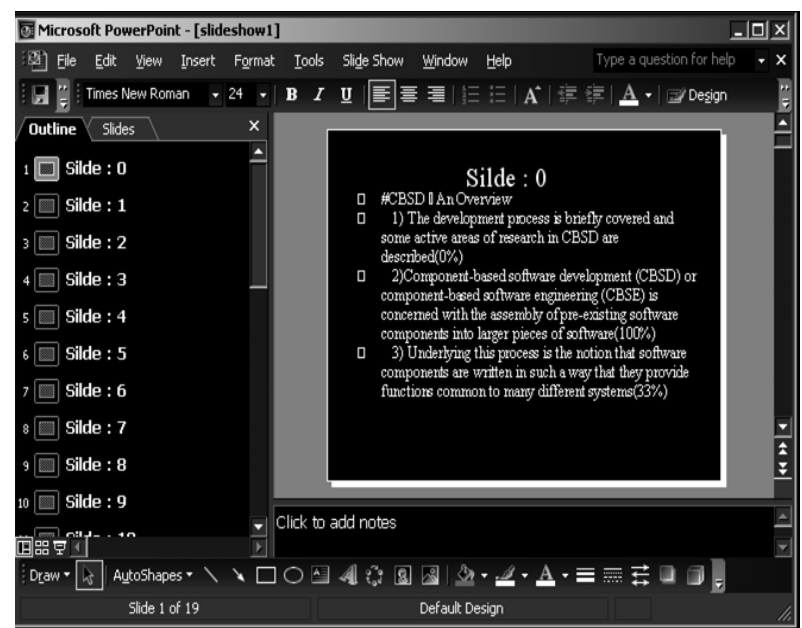

Fig.17. PPT generated by user1 for the CBSD topic

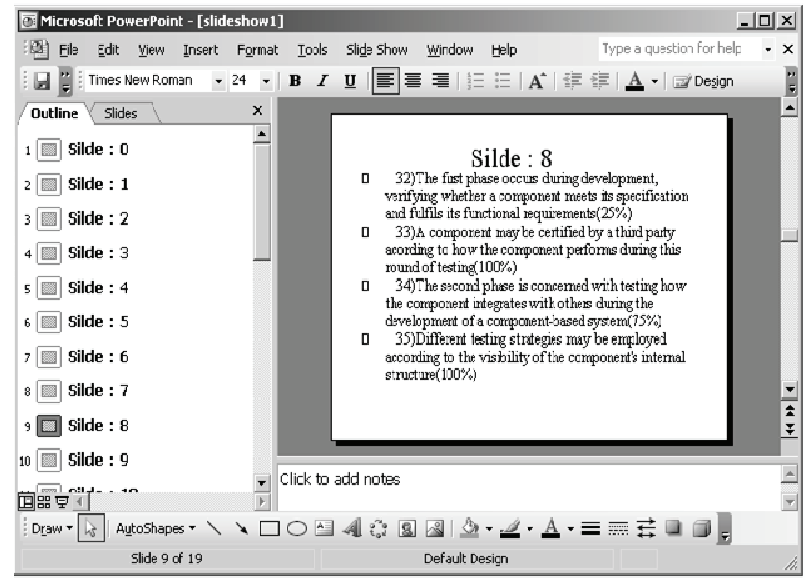

Fig.18. PPT generated by user2 for the CBSD topic

\section{CONCLUSION}

The presentation is generated for the textual data that are present in the documents. The images are not present in the documents which are taken for the generation of PPT. In future, the presentation can be enhanced by embedding the images, animations and sound.

\section{REFERENCES}

[1] Robert M. Losee, June 1998, "Comparing Boolean and Probabilistic Information Retrieval Systems Across Queries and Disciplines". Journal of the American Society for Information Science

[2] Manning, Raghavan, and Schutze, 2007, "Introduction to Information Retrieval", Cambridge University Press, pp.116 , pp.169-182

[3] Kuang-hua Chen and Hsin-Hsi Chen, 1995, "A Corpus Corpus Based Approach to Text Partition", Proceedings of the Workshop of Recent Advances in Natural Language Processing, pp. 152-161 
[4] Han-joon Kima, Sang-goo Lee, 2003, "Building topic hierarchy based on fuzzy relations", Elsevier publications of Neuron computing, pp.481-486

[5] Youngjoong Ko, Jinwoo Park, Jungyun Seo, 2004, "Improving text categorization using the importance of sentences", Elsevier publications of an Information Processing and Management, pp.65-79.

[6] White, R. W., Jose, J. M., Ruthven, 2005, "Using top ranking sentences to facilitate effective information access", JASIST, Vol. 56, No. 10, pp.1113-1125

[7] Hyo-Jung Oh, Sung Hyon Myaeng, Myung-Gil Jang, 2007, "Semantic passage segmentation based on sentence topics for question answering", Elsevier publications of Information Sciences, pp.3696-3717.
[8] Shailendra Singh, Lipika Dey, 2005, “A rough-fuzzy document grading system for customized text information retrieval", Elsevier publications of Information Processing and Management, pp.195-216

[9] Xiao luo, A. Nur zincir- Heywood, 2004, “Combining Word Based and Word Co-Occurrence Based Sequence Analysis For Text Categorization", Proceedings of the Third International Conference on Machine Learning and Cybernetics, pp.1580-1585.

[10] Softpedia, free-to-try software programs, http://www.softpedia.com/get/Others/HomeEducation/Wintree.shtml. 